\title{
Design of a Smart Stick Prototype Using Goal Oriented Requirements Engineering Methodology
}

\author{
Vinay S \\ Research Scholar, Manipal University \\ Asst Prof, NMAMIT, Nitte, Karnataka \\ India
}

\author{
Niha Noor Shaikh \\ Student, MSc Software Engineering \\ University of Southampton, \\ United Kingdom
}

\author{
Sridhar Aithal \\ Professor, Research Guide, Manipal \\ University, Manipal, Karnataka \\ India
}

\begin{abstract}
The primary purpose of this project is to assist the visually challenged (user) in shopping. The project makes use of GORE (Goal oriented Requirements Engineering Methodology). The device developed is based on Radio Frequency Identification (RFID) which operates in the Low Frequency (LF) band. The envisioned device is a combination of a RFID LF reader module and a microcontroller unit to convey all the information pertaining to the product to the user, thereby enhancing their shopping experience.
\end{abstract}

\section{Categories and Subject Descriptors}

D.2.1 [Requirements/Specifications]: Methodologies, Tools D.2.10 [Design]: Methodologies

\section{General Terms}

Algorithms, Design, Economics, Experimentation, Human Factors, Languages.

\section{Keywords}

Radio Frequency Identification (RFID), Low Frequency (LF), Shopping Assistants, Visually Challenged, KAOS, GORE

\section{INTRODUCTION}

Of the 37 million people across the globe who are blind, over 15 million are from India [1]. So, it is rather unfortunate that there aren't many friendly environments (parks, shopping malls, libraries) for the visually challenged. RFID is a very versatile technology which is being used in several avenues such as the retail industry, libraries and asset tracking [2].

A RFID system consists of 3 main components, RFID reader comprising an antenna and digital signal processor; tags or transponders comprising an integrated circuit and an antenna and finally the middleware or software application to process the data read by the reader from the tag.

\section{RELATEDWORK}

Vladimir Kulyukin et al have worked on a prototype, ShopTalk that demonstrates the proof of concept wherein the visually challenged can find shelved products in grocery store [3]. Here, it is already assumed that verbal route directions and layout description is available. In several other research and experiments carried out by the aforementioned individuals in the same domain, more emphasis is laid on navigation using Radio Frequency (RF) technology. However, in ShopTalk products are identified using barcodes and wireless barcode scanners are facilitated rather than RFID tags and readers.

While barcode scanners are still being used, the same method to help the visually challenged shop is not suitable as it still requires line of sight. Hence, in this paper we have proposed the replacement of barcode scanners at the billing counter with LF RFID reader which will not only speed up the billing process but also assist the visually challenged to shop independently. The working of an analysis prototype to achieve the same is presented.

\section{OBJECTIVE}

The primary objective is to enable the visually challenged to shop independently. It is better that the supermarkets implement a system wherein the products are tagged using LF tags. Each LF tag has a unique number assigned by the manufacturer which is in turn assigned to the barcode of the respective product. RFID technology is chosen because it doesn't require line of sight which is an added bonus and satisfies the basic requirements of our application.

There are a number of manufacturers of Ultra High Frequency (UHF) products. But there is no standardization with respect to bandwidth utilization (800-900 MHz) in the industry. LF RFID uses the $125 \mathrm{KHz}$ frequency. Also, the initial investment is very low. Hence LF RFID offers a solution better than the existing ones. To realize this objective, an analysis prototype has been implemented.

\section{PROPOSED SYSTEM}

The proposed device is compact and portable which can be attached or detached from the cane (used by the visually challenged) at any time. It consists of the following:

1) Keys numbered 1-6 and an alphabet " $b$ " in Braille.

2) A button/switch to activate/deactivate the device.

3) A button/switch to activate/deactivate Bluetooth.

4) A built-in microphone or a jack for external microphone support.

5) A jack for external headset support

6) A Universal Serial Bus (USB) port for external pen drive support.

7) A wireless LF RFID reader.

8) A microcontroller to handle all processing logic. 
9) Power unit with charger

The following assumptions were kept in mind while developing the prototype,

1) The supermarket follows standard rack specification and layout.

2) The supermarket database is sorted under the device applications predefined item categories.

\section{METHODOLOGY}

To realize the objectives stated in Section 3, we have employed KAOS - A Goal Oriented Requirements Engineering methodology (GORE). GORE approach attempts to solve some of the inadequacies of the traditional systems analysis approaches ([4],[5],[6]) while dealing with more and more complex software systems. Some of the benefits of goal modeling include [7]: them.

Goals provide rationale for requirements that operationalize

Goals provide a precise criterion for sufficient completeness of a requirements specification.

Goal models provide an excellent way to communicate requirements to customers. Goal refinements offer the right level of abstraction to involve decision makers for validating choices being made among alternatives and for suggesting other alternatives

The KAOS methodology is a goal-oriented requirements engineering approach with a rich set of formal analysis techniques. KAOS stands for Knowledge Acquisition in automated Specification [8] or Keep All Objects Satisfied [9]. KAOS is described in [9] as a multi paradigm framework that allows to combine different levels of expression and reasoning: semi-formal for modeling and structuring goals, qualitative for selection among the alternatives, and formal, when needed, for more accurate reasoning.

We briefly discuss the use of this methodology for a specific scenario. KAOS makes use of generic requirements patterns and we have used one such pattern for building a cheap system as shown in the figure 1 . Since we had to design a system keeping the cost of the final product in mind we had to analyze the alternatives for realizing our goal of building a cheap and efficient system.

KAOS methodology generates 4 models: goal model, responsibility model, object model and operation model. The models generated will come in handy to implement the design in any object oriented language with the help of UML diagrams.

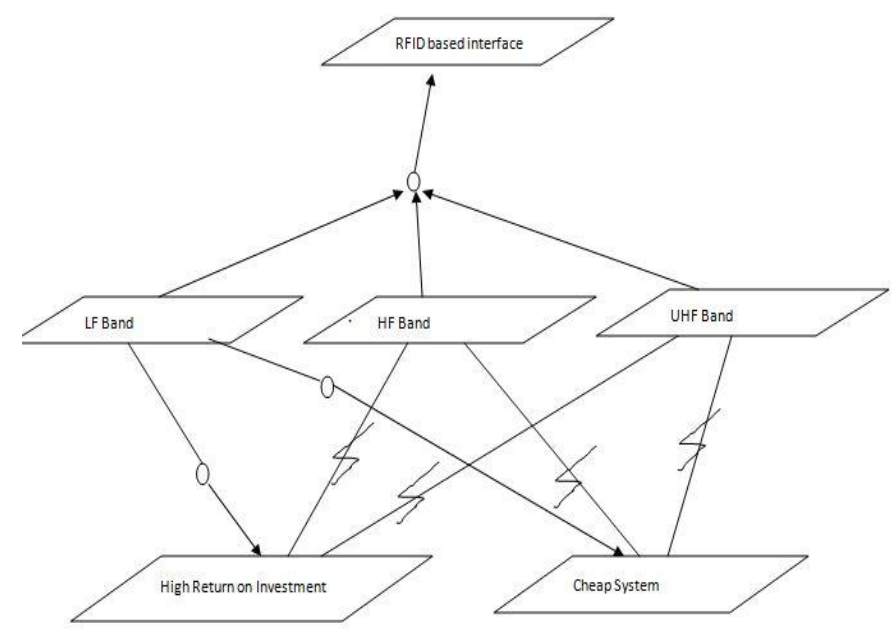

Figure 1: Analyzing alternatives using KAOS methodology

Figure 1 represents a scenario where in to realize the goal of RFID based interface for our system, we have 3 alternatives: Low frequency (LF) band, High frequency (HF) band and Ultra High frequency (UHF) band. Considering the cost factor, both HF and UHF conflicts with the non functional goals: cheap system and high return on investment. Whereas a LF based implementation has a positive impact on both the non functional goals. This quantitative and qualitative evaluation of alternatives is an important feature of KAOS methodology and we have made extensive use of it in designing our system.

More information on KAOS and Objectiver - a tool designed to support KAOS can be had from [10].

The basic operating procedure of the prototype illustrated in the Figure 2 is as follows,

1) The pen drive that contains the supermarket data file is handed over to the user. Upon receiving this, the pen drive is plugged onto the prototype.

2) On picking any item, the RFID reader activates the RFID tag(within a range of $9 \mathrm{cms}$ ) which sends the unique manufacturer assigned ID to the reader.

3) The prototype reads the tag ID from the serial port and sends a query to the data file on the pen drive.

4) The retrieved details are converted to speech and can be played back to the user via Bluetooth/wired headsets. 


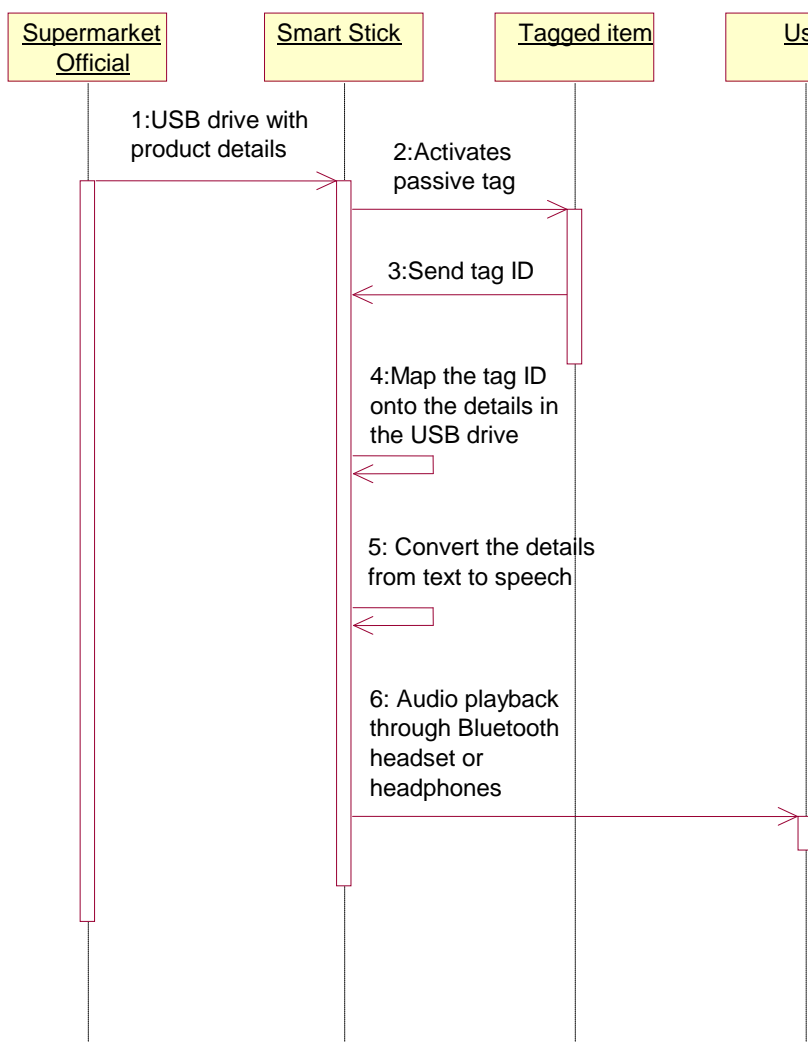

Figure 2: Sequence diagram depicting the working of the prototype

\section{DESIGN AND IMPLEMENTATION}

The application (prototype) is designed to provide a lot of flexibility to the user. All the options are conveyed to the user by synthesizing text to speech. The first phase of the application is an interactive menu providing the following features to the user.

\subsection{Pick Products}

This feature is available to the user by pressing key 1(option 1). This enables the RFID reader and all details pertaining to the product will be conveyed to the user via voice output.

\subsection{Pick Products with Speech Recognition}

\section{Enabled}

This feature is available to the user by pressing key 2(option 2). This can be used to query beforehand to check the availability of the product. For example, if the user says "Kellogg's Corn Flakes", he will be informed if it is available along with the prices of the packaging. The function of Pick Products is also available under this option.

\subsection{Enunciated Location of Item \\ Categories}

Enunciated location of Item Categories has two options: option 3(enunciation and voice commands) and option 4(enunciation and keyed commands). The application provides a list of three predefined main categories (Food, Beverages, Toiletries). The aisle number of each main category and sub category is enunciated to the user, thereby facilitating location of the product.

In option 3 the main category names are enunciated (option 3 ) and the user has to select the category by voice commands. The submenus (sub categories) are then enunciated and again the selection is by voice commands to obtain the location.

Under option 4, numbers are assigned to each main category and selection of the main categories has to be keyed. Similarly the submenus (sub categories) also have a similar provision.

\subsection{Hear Last Five Tags}

This feature is available to the user by pressing key 5(option 5).This allows the user to replay the information related to the last 5 tagged products he picked.

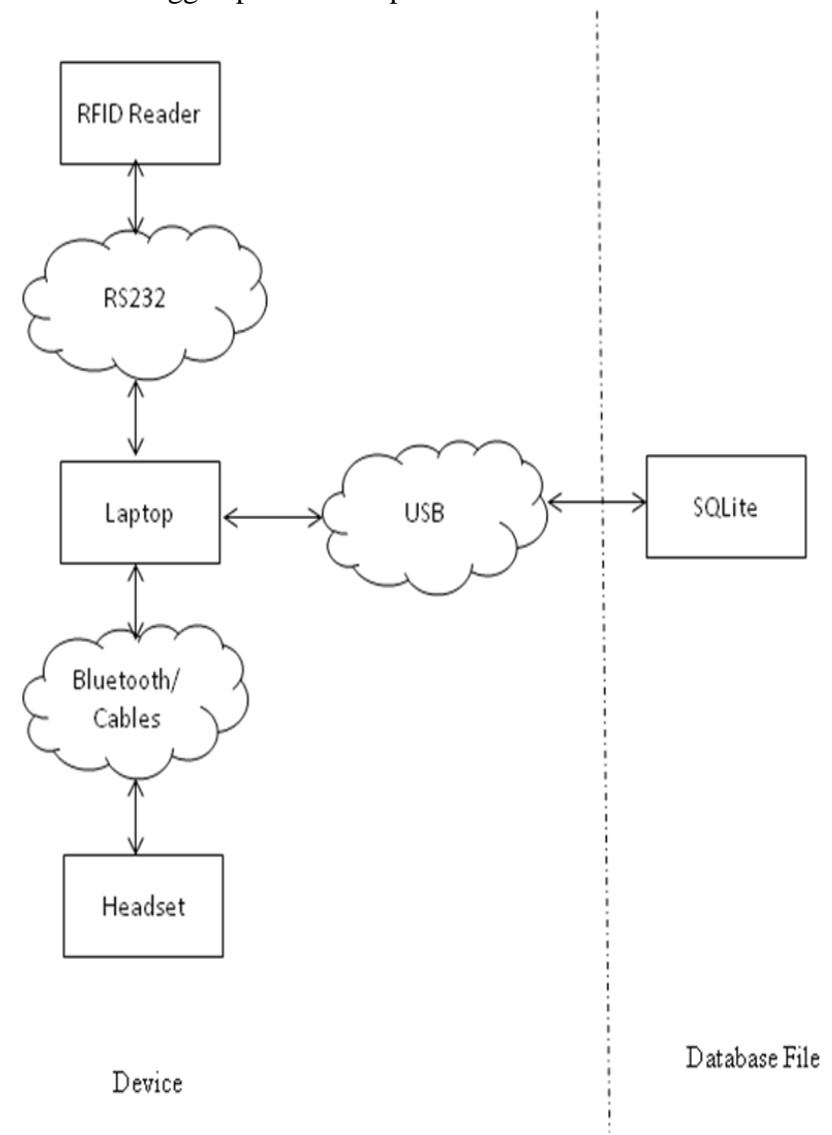

Figure 3: Prototype perspective

The figure 3 describes the prototype and its interfaces as follows,

1) The RFID reader is interfaced with the laptop using a serial cable via USB-Serial converter cable. The RS-232 standard is used. 
2) The headset to hear the speech synthesis of product details can be interfaced via Bluetooth or cables with the laptop.

3) SQLite data file resides on the pen drive and is interfaced with the laptop via USB port.

The unique key design feature is the access of the database from the pen drive instead of having a Bluetooth communication channel between the prototype and the point of sale to map the ID and get the product details, thereby giving a good response time.

\section{FINDINGS AND ANALYSIS}

For the purpose of our prototype which demonstrates proof of concept, the capability of the device is being demonstrated on a Toshiba Satellite A100-036 laptop.

The .NET Framework by Microsoft with the development language as $\mathrm{C \#}$ was chosen since this is an event driven application involving data access [11].

Microsoft also provides Speech APIs which can be seamlessly integrated with the applications developed within this framework.

Atmel's ARM9 microcontroller comes preloaded with Windows CE $[12,13]$. For the envisioned device, such a microcontroller provides more flexibility and power to the implementation of the application.

The simulated environment has implemented item level tagging.

The prototype can easily give the audio output for the products picked up by the user.

Dictation based voice recognition becomes rather erratic in extremely noisy environments. However, training the profile in the noisy environment has increased the rate of accuracy marginally.

Command based voice recognition works accurately with any user's input and doesn't require extensive training as the aforementioned.

The RFID reader is a low frequency one operating in the band 125 $\mathrm{KHz}$ which can read a tag from $9 \mathrm{cms}$ approximately. To increase the range, the module provides pins for external antenna connection. The new range with additional antennas is yet to be tested. This will be required to test the prototype in a rack level tagging method.

The module is connected via DB9-RS232 pin and the tag ID is directly read from the serial port. The HyperTerminal can be used to configure the Baud Rate, Parity, Data Bits and the Stop Bit but we have directly initialized the same in our .NET C\# console application.

Middleware is not used because the application requirements do not demand its usage as the amount of data generated can be easily handled by directly accessing the serial port.

The prototype, called RG, is a robotic shopping assistant for the blind which is a brainchild of Vladimir Kulyukin , an assistant computer science professor at Utah State costs $\$ 5000$ and still has a long way to go before it is marketed and mass produced[14]. On the contrary, our aforementioned device is comparatively commercially viable. For the prototype, the RFID reader module costs $\$ 50$ and each LF tag costs about 90 cents. This can work out ever cheaper when one considers the proposed device. Further, Atmel's ARM9 AT91SAM9260 microcontrollers is priced at $\$ 6$ in volumes of $100 \mathrm{k}$ units $[12$, 13].

\section{CONCLUSION AND FUTURE ENHANCEMENTS}

Smart Stick is still a very basic prototype with a lot of potential and scope for improvements as it is based on a very versatile technology: RFID. Currently the following areas can be worked and improved upon.

The navigation feature can be added to the device wherein the user is guided to the aisle of his choice or is informed when he steps into a new aisle. Talking Points can be used for this purpose. Talking Points provide contextual information of what has been on the path taken by the user to reach from point $A$ to point $B$ and they make use of Bluetooth beacons which would be located at points of interest from where the owner wishes to share any information with Talking Points users [15, 16, 17].

The obstacle detection module should be able to detect obstacles and notify user with beeps that vary in frequency depending on the distance of the obstacle.

The range of obstacle detection should be adjustable by the user based on some options. For instance, if 10 meter range may work in open areas like roads and gardens, it may not work well inside a supermarket. So the user must be able to speak to the device to set its range based on the current environment.

The device must have the capability to process the data file and convert it to the layout it supports if it is not already following the same. For example, if the item categories in the database are not as per the device specifications then the process of sorting and processing the database contents into the predefined categories should be possible.

The user must be able to create his shopping list via speech recognition. This will enable him to get the brands for those items and their location.

Support for functioning in haphazard supermarket layouts can also be added.

Voice training for speech recognition is currently not a part of the device application. It can be integrated in the device application where the user is prompted to train the system before using it.

Speech version of product details can be supported in various languages apart from English.

A RFID based analysis prototype was developed to serve as a shopping assistant for the visually challenged users. Findings and analysis shows that the prototype has successfully demonstrated the proof of concept where major emphasis and stress lays on relaying product details when the user picks an item in the supermarket. We have started our work towards developing the envisaged product. 


\section{ACKNOWLEDGMENTS}

We acknowledge our sincere thanks to Prof. K M Hebbar, Prof. Niranjan Chiplunkar, Prof. Stephan Vadakkan and Prof Ashok Kumar for all the help and insight provided during this project.

\section{REFERENCES}

[1] India has largest blind population [Article]/ auth. Sinha Kounteya // The Times of India. - October 11, 2007.

[2] Common Applications: Using RFID for Asset Tracking and Other Applications [Online] // RFID Labeling. http://www.rfidlabeling.com/commonapplications.html

[3] ShopTalk: Toward Independent Shopping by People with Visual Impairments[Conference]/ auth. Kulyukin Vladimir, Nicholson John and Coster Daniel. - 2008.

[4] D. Ross. Structured Analysis: A Language for Communicating Ideas. IEEE Transactions on Software Engineering, 3(1), January 1977.

[5] T. DeMarco. Structured Analysis and System Specification. Yourdon Press. 1978.

[6] J. Rumbaugh, M. Blaha, W. Premerlani, F. Eddy, W. Lorensen. Object-Oriented Modeling and Design. Prentice Hall. 1991.

[7] A. van Lamsweerde. Goal-Oriented Requirements Engineering: A Guided Tour. Proc. 5th IEEE International Symposium on Requirements Engineering (RE'01), Toronto, Canada, August 2001.

[8] A. Dardenne, A. van Lamsweerde and S. Fickas. GoalDirected Requirements Acquisition. Science of Computer Programming, 20(1-2), April 1993.
[9] A. van Lamsweerde, E. Letier. From Object Orientation to Goal Orientation: A Paradigm Shift for Requirements Engineering. Proc. Radical Innovations of Software and Systems Engineering, LNCS, 2003.

[10] Objectiver tool - http://www.objectiver.com/

[11] Blind-Accessible Shopping System Design / auth. Becker David [et al.]. - 2006.

[12] ARM9 microcontroller costs \$6, runs Windows CE [Online] // Windows for Devices. - June 8, 2006. http://www.windowsfordevices.com/news/NS5994380097.html

[13] Windows Embedded CE Overview [Online] // MIcrosoft. http://www.microsoft.com/windowsembedded/en-

us/products/windowsce/default.mspx

[14] Robot lends 'a seeing eye' for blind shoppers [Online] / auth. Burrell Ashley // USA Today. - November 7, 2005. http://www.usatoday.com/tech/news/techinnovations/2005-07-11robot-guide_x.htm

[15] New Bluetooth system orients blind and sighted pedestrians [Online]. - $\quad$ September $\quad 18, \quad$ 2008. http://www.ns.umich.edu/htdocs/releases/story.php?id=6737

[16] University of Michigan students develop system to help tourists wandering cityscapes [Online] / auth. Gershman Dave // Ann Arbor Living. - October 13, 2008. - University of Michigan students develop system to help tourists wandering cityscapes. [17] Audio Reality: Bluetooth for the Blind [Online]/ auth. Jordan // NowPublic News Coverage. - September 19, 2008. http://www.nowpublic.com/tech-biz/audio-reality-bluetoothblind. 